University of New Mexico

UNM Digital Repository

10-1-1974

\title{
Hunting by expectation or optimal foraging: A study of patch use by chickadees
}

John Krebs

John Ryan

Eric Charnov

Follow this and additional works at: https://digitalrepository.unm.edu/biol_fsp

Part of the Biology Commons

\section{Recommended Citation}

Krebs, J. R., J. Ryan and E. L. Charnov. 1974. Hunting by expectation or optimal foraging: A study of patch use by chickadees. Animal Behaviour 22:953-964

This Article is brought to you for free and open access by the Academic Department Resources at UNM Digital Repository. It has been accepted for inclusion in Biology Faculty \& Staff Publications by an authorized administrator of UNM Digital Repository. For more information, please contact disc@unm.edu. 
Anim. Behav., 1974, 22, 953-964

\title{
HUNTING BY EXPECTATION OR OPTIMAL FORAGING? A STUDY OF PATCH USE BY CHICKADEES
}

\author{
BY JOHN R. KREBS, * JOHN C. RYAN \& ERIC L. CHARNOV $\dagger$ \\ Institute of Animal Resource Ecology, University of British Columbia, Vancouver 8
}

\begin{abstract}
When a predator exploits an environment in which food is patchily distributed, it has to continually make the decision of how long to stay in a patch. In this paper we examine this question using captive black-capped chickadees foraging in a large aviary for small pieces of mealworm hidden in artificial pine cones. The results of our experiments show the following: (a) when the birds encounter a long sequence of patches (groups of pine cones) each containing the same number of prey, they do not learn to expect a fixed number of prey per patch (Gibb's hypothesis of hunting by exfectation); (b) similarly, the birds do not learn to spend constant time on each patch (although the data on this are less clear cut); (c) the birds have a constant giving-up time (interval between last catch and leaving the patch) for all types of patches within an environment, and the giving-up time is inversely related to the average capture rate for the environment. These two findings are consistent with an optimal foraging model of patch use developed by E. L. Charnov. Thus our results are more consistent with the predictions of the optimal foraging than with the hypothesis of hunting by expectation.
\end{abstract}

In this paper we describe a study designed to look at part of the general question 'How does a predator exploit an environment in which food is patchily distributed?' We are concerned with the type of predator whose food occurs in more or less discrete clumps (or patches) and we assume that the predator has to visit many such patches during the course of a single day's foraging. This type of predator is potentially confronted with two decisions concerned with the efficient exploitation of a patchy environment, although the two may resolve into one. The first decision stems from the fact that patches are unlikely to be of equal worth, and the efficient predator must choose patches of high profitability (MacArthur \& Pianka 1966; Royama 1970; Tullock 1971). If the predator could actually see the contents of each patch (e.g. berries on a bush), then this initial choice could be made on the basis of visual scanning. Alternatively, if patches are to some extent predictable and contain a type of food that is continuously renewing itself, the predator might learn by experience which are the most profitable patches (Smith \& Dawkins 1971; Simons \& Alcock 1971). The second decision confronting the predator, which we investigate in this paper, is at what point to leave one patch and go on to the next one. The efficient predator has to *Present address: Department of Zoology, South Parks
Road, Oxford OX1 3PS. $\dagger$ Present address: Department of Biology, University
of Utah, Salt Lake City, Utah, 84112, U.S.A. estimate when it is no longer worthwhile scouring around in the present patch. If for any reason the quality of patches cannot be predicted in advance (as must often be the case in nature) then the first decision becomes congruent with the second, and the predator exploits patches by sampling them as they are encountered and deciding whether or not to stay. We have tried to answer the question 'On what criteria does a predator decide to leave a patch?' Our predator is the black-capped chickadee Parus atricapillus.

The starting point for our study was the work of Gibb (1958, 1962a, b, 1966), who carried out a detailed field study into the way in which titmice (Parus spp.) exploit the larvae of a eucosmid moth Ernarmonia conicolana that lives in the cones of Scots pine (Pinus sylvestris) during the winter months. Gibb was able to reconstruct the pattern of predation by the birds on the caterpillars because the tits hack open the cones to get at the hidden larvae, and thus leave evidence of successful predation. Larvae still surviving at the end of the winter can be found by dissection of the cones. Gibb thought that the birds detected the larvae either by tapping on the cones or by looking for minute visual cues. By collecting cones, Gibb could measure the initial number of larvae present per group of cones (the intensity) and the number eaten by the birds, and thus calculate the proportion of larvae eaten. From our present point of view, the crucial evidence was this: in some areas of uniformly low intensity there were occasional pockets of high-intensity cones, 
for example on a heavily infested tree, and in these pockets, the birds took proportionately fewer larvae than from the surrounding lowintensity areas (Gibb 1962a, b). Gibb explained this observation by suggesting that the birds were learning to expect a certain number of larvae in each cone or group of cones, and that having found the expected number, the bird would leave a patch (group of cones) and pass on to the next one. Thus if the patch was a particularly high-intensity one in relation to surrounding patches, the birds took proportionately fewer caterpillars. Gibb's hypothesis of 'hunting by expectation', put forward in this specific context, could conceivably be a general hypothesis to account for the way predators use a patchy environment containing hidden prey.

However, the data on which Gibb based his idea are, to say the least, somewhat equivocal. Firstly, very few instances of hunting by expectation were observed, and secondly the observations that were made did not clearly support Gibb's idea (Simons \& Alcock 1971; Krebs 1973). A further point is that Gibb's mechanism would only work if the spatial distribution of prey were such that most patches contained a standard number of prey (otherwise the bird would not be able to form an expectation). As an aside, it is worth mentioning that Gibb's data for the broader scale pattern of predation between different parts of his study area showed intensity dependent predation rather than the negatively intensity dependent predation predicted by the hunting by expectation hypothesis. Of course, the broad scale pattern of predation does not necessarily tell us how individual birds were behaving on a minute to minute basis.

Our study initially set out to test the idea of hunting by expectation, experimentally using captive chickadees. However, at the same time we also examined two alternative hypotheses. The first of these is a modification of Gibb's idea. Krebs (1973) suggested that the birds in Gibb's study might have formed a time expectation rather than a number expectation, i.e. that the birds learned to spend a constant amount of time on each cone or cone group rather than looking for a constant number of prey. This idea has the advantage that animals are on the whole better at measuring time than at counting. If the birds did follow a time expectation, the number of prey taken per patch would follow Holling's disc equation
(Holling 1959) as shown by Krebs (1973). This would produce a result compatible with Gibb's observations.

The third hypothesis of patch use is based on an optimal foraging model (Charnov 1973). The model, which is published in full in Charnov \& Orians (in press), asks what strategy a predator should employ in using a patchy environment in order to maximize its net rate of food intake while foraging (see also Emlen 1973). The long-term goal of an optimal forager is obviously to maximize its fitness, but we use food intake, which we presume is related to fitness, as the short-term goal. The model is presented graphically in Fig. 1 and below in a simplified algebraic form. In essence the model assumes that the capture rate within a patch decreases with time spent searching (because of depletion) and that when the capture rate drops to a critical level the predator leaves. The problem is to find this critical level.

Suppose that we have an environment made up of two patch types. A bird hunts on a patch and spends time in travelling between patches, the density of patches is such that $t$ is the average travel time between them and $P_{\mathrm{A}}$ or $P_{\mathrm{B}}$ is the proportion visited that are of type $A$ or $B$. While in a particular patch, the bird reduces the food density such that the within-patch capture rate is a decreasing function of the time spent in the patch. The total amount of food taken from a patch $(f(T))$ thus rises from zero at a negatively accelerated rate (with time spent in the patch, $T$ ) to an asymptote represented by the total amount of food present in the patch at the start. Let $T_{\mathrm{A}}$ denote the time spent in a patch of type A. ( $T_{\mathrm{B}}$ for B). The average time to use one patch is the travel time plus the time spent in the patch.

$$
T=t+P_{\mathrm{A}} \cdot T_{\mathrm{A}}+P_{\mathrm{B}} \cdot T_{\mathrm{B}}
$$

The average amount of food taken from one patch is

$$
E=P_{\mathrm{A}} \cdot f\left(T_{\mathrm{A}}\right)+P_{\mathrm{B}} \cdot f\left(T_{\mathrm{B}}\right)
$$

$E / T$ is an average rate of food intake, and we wish to maximize it in the choice of $T_{\mathrm{A}}$ and $T_{\mathrm{B}}$. To do this we set.

$$
\delta(E / T) / \delta T_{\mathrm{A} \text { or B }}=0
$$

This gives a rule that $\delta(E / T) / \delta T_{i}=0$ when

$$
\frac{\delta f\left(T_{i}\right)}{\delta T_{i}}=E / T
$$

(the same for all patch types in the habitat.) 
Equation (4) says that the predator should leave the patch when its rate of food intake in the patch drops to the average rate for the habitat, and that this marginal capture rate should be equalized over all patches within a habitat. If the prey are all the same size and have

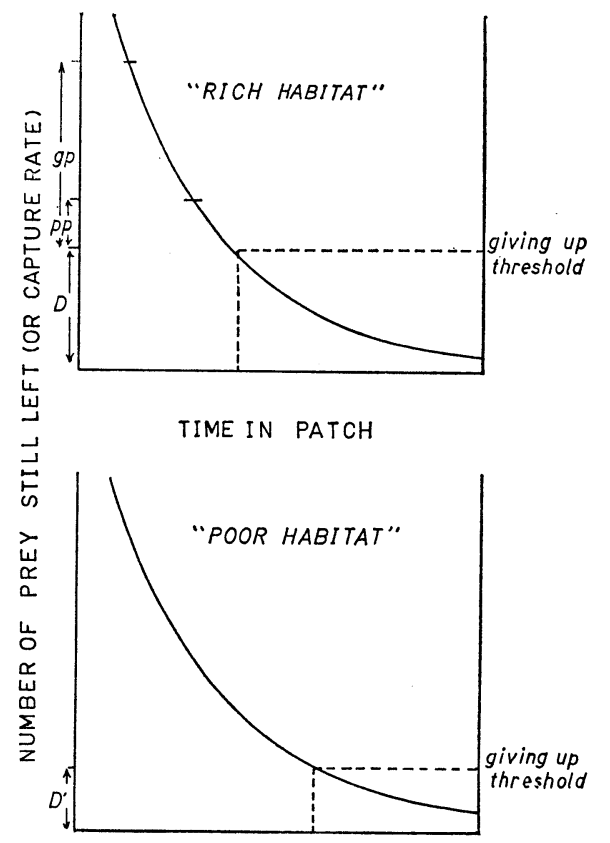

Fig. 1. A graphical representation of an optimal foraging model of patch use. The upper and lower graphs are similar except that the former refers to a 'rich habitat', where the average capture rate is high, and the latter to a 'poor habitat'. The graphs show the number of prey surviving in a patch plotted against time spent by the predator searching in the patch. The shape of this survivorship curve is not specified by the model, other than the decrease with time. If we assume that the capture rate within a patch is proportional to the number of prey present, the two curves show the change in capture rate with time spent searching in a patch. The predator leaves a patch when its capture rate drops to a critical level, called the 'giving-up' threshold on the graphs. The model predicts that this giving-up threshold is equal to the average rate of food intake for the habitat. The givingup threshold is higher in the rich habitat (upper graph) than in the poor. We measured the giving-up threshold in our experiments by measuring the giving-up time. The giving-up time is inversely proportional to the number of prey surviving after the predator leaves $(D)$. The giving-up time is shorter in the rich than in the poor habitat $\left(1 / D<1 / D^{\prime}\right)$. The numbers of prey taken from good and bad patches within the rich habitat are indicated by the lines $g p$ and $p p$ respectively. We have assumed for the sake of simplicity that the survivorship curves for good and bad patches are similar, but that a bad patch starts lower down on the curve. small handling times (as in our experiments), the rate of food intake is approximately equal to the reciprocal of the intercatch interval, and we can substitute time for rate of food intake in the predictions of the model. The predictions of equation (4) then become (a) the predator should have a constant 'giving-up time' (Croze 1970) for all patch types within a habitat, the giving-up time being the interval between the the last catch and leaving, (b) giving-up time should be shorter in better habitats, where the average capture rate is higher. More precisely, the ratio of giving-up times in two habitats should be equal to the ratio of the mean capture rates for the two environments; (we use the terms habitat and environment interchangeably in this paper).

The derivation of equation (3) is simplified, and the actual relationship shown in (4) is much more general than given here. To show this requires results from renewal theory and will be published elsewhere (Charnov \& Orians in press).

\section{Methods}

The tests were carried out using six, hand-raised, black-capped chickadees (Parus atricapillus). The birds were taken from the nest at the age of about 10 days and raised on the diet described by Krebs, MacRoberts \& Cullen (1972). The birds were housed in a large aviary (Fig. 2) and one individual at a time was taken from the holding aviary for the experiments. The tests were carried out between October 1972 and June 1973.

The test aviary was divided into two sections, indoor and outdoor, connected by a trap door. The tests were run in the indoor section and while a test was being set up inside, the test bird was shut outside by closing the trapdoor. At the start of a test, the trapdoor was opened and the bird flew in immediately; during a test the bird could fly freely between the indoor and outdoor sections.

The experimental set up (Plate $\mathrm{XV}(\mathrm{b})$ ) was based on Gibb's study of tits hunting for caterpillars in pine cones. It consisted of five artificial 'trees' (Fig. 3) with 'pine cones' (small blocks of wood with three holes drilled on each of two adjacent sides) hung in groups of four from the branches; the cones were arranged so that they could be removed between tests. Each tree had three groups of four cones; thus the whole area contained sixty cones in fifteen groups. The cones were arranged in groups 


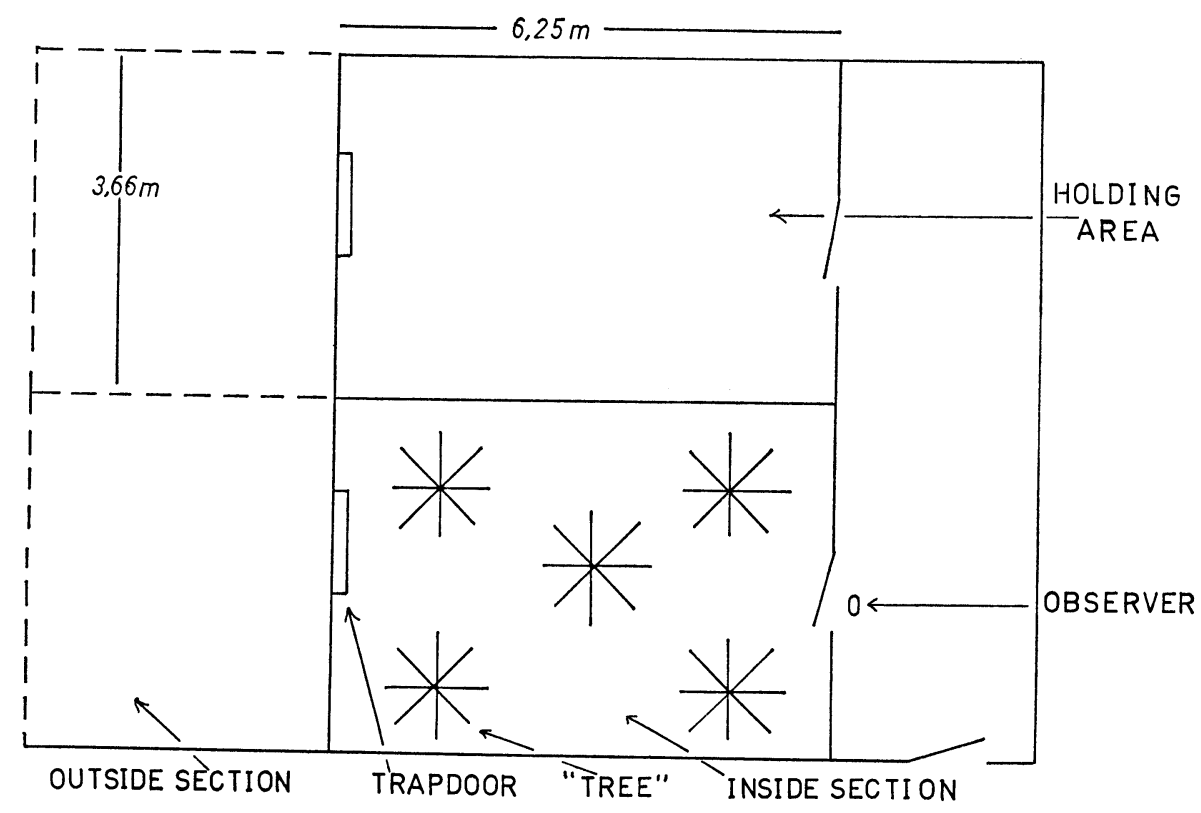

Fig. 2. A plan view of the experimental aviaries.

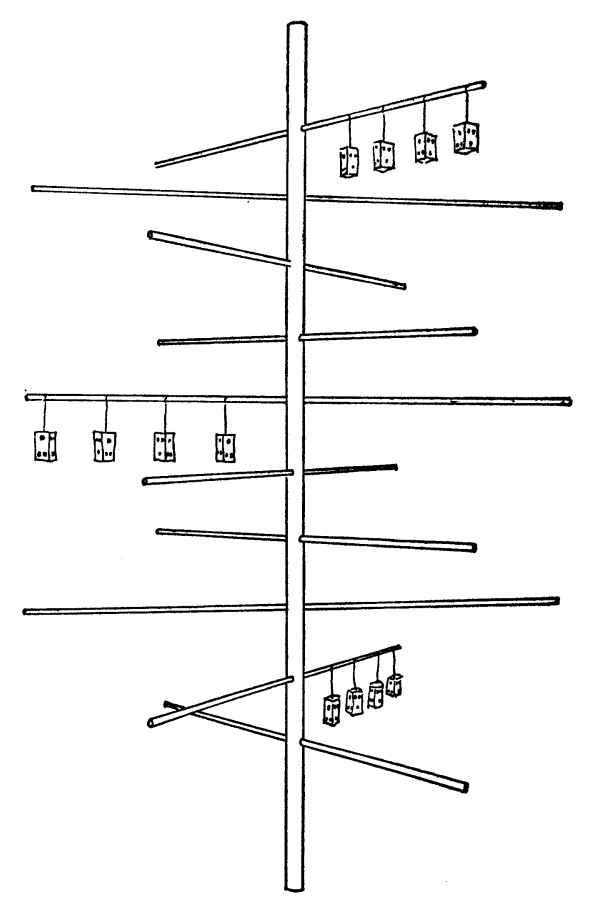

Fig. 3. An experimental tree with artificial cones hanging in groups. because this is the way they occur in nature, and because Gibb's data applied not to individual cones but to groups of cones. We did not know whether the birds would treat cones or groups as 'patches' but looked for both possibilities in the data. All the birds very readily learned to search on the cones by hanging on a cone and peeling off the white sticky labels that were used to cover the holes (Plate XV(a)). Although black-capped chickadees do not regularly forage in pine cones for hidden larvae, they do use similar foraging tactics in other contexts. Ryan (1973), for example, reports that they peck open galls of the thimble berry gall fly (Diastrophus kincaidii) in a way that must be similar to the foraging of Gibb's titmice on pine cones. The rewards in the holes of the artificial cones were pieces of mealworm weighing approximately $0.036 \mathrm{~g}$, which were small enough for the bird to eat immediately without leaving the cone. The tests lasted about 5 min each, a short enough time for depletion effects not to be a major consideration. Between each test, the bird was shut outside and the cones and stickers replenished as necessary. Usually not more than three tests were run in any one day.

During the tests the bird's behaviour was recorded on a Dawkins' behaviour organ 
Table I. Experiment 1. Number of Mealworm Pieces Found (a) Per Cone and (b) Per Cone Group in Type-one Tests (Low Intensity Cones) and in High Intensity Cones of Type-two Tests. Data for Six Birds Averaged

\begin{tabular}{lccc}
\hline & $\begin{array}{c}\text { Low intensity } \\
\text { mean }(N=6) \mathrm{SD}\end{array}$ & $\begin{array}{c}\text { High intensity } \\
\text { mean }(N=6) \mathrm{SD}\end{array}$ & $t$ \\
\hline (a) No. of larvae found per cone & $0.235 \pm 0.03$ & $0.52 \pm 0.03$ & $6.25^{*}$ \\
(b) No. of larvae found per group & $0.72 \pm 0.11$ & $1.87 \pm 0.40$ & $16.74^{*}$ \\
\hline
\end{tabular}

$* P<0.01$

(Dawkins 1972) and the data were subsequently fed into the computer for analysis. The following events were recorded: (i) arrival on a particular tree; (ii) arrival on a cone group; (iii) arrival on a cone; (iv) look (bird peels off a sticker and peers into hole); (v) find (bird finds mealworm piece); (vi) leave (bird leaves a cone, cone group, or tree); (vii) switch (bird switches to next cone in the same group); (viii) return (bird returns to same cone).

In the first experiment (see below) individual cone groups were not identified, so re-visiting could not be distinguished from first-time visits. In the second experiment, individual groups were identified. The procedural details for the two different experiments are described separately under the two sections dealing with experiments. The data were analysed statistically using, where appropriate, paired or unpaired $t$-tests and oneway analysis of variance.

\section{Experiment 1}

This experiment was particularly designed to test the hypothesis of hunting by expectation. The idea was to give the birds a lot of experience with 'low-intensity cone groups' (one mealworm piece per cone, four per group), and relatively rare encounters with 'high-intensity cone groups' (3 larvae per cone, 12 per group). In the wild this situation would presumably occur, as in Gibb's study, if the birds were foraging in a large area of lightly infested trees and then suddenly encountered one or two heavily infested trees. In our experiment the birds encountered low-intensity trees in a succession of short tests in the same area, rather than by wandering over a large area, as in the wild. We have to assume that this difference does not influence the way in which the birds search.

\section{Procedure}

The birds were first given a training period consisting of twelve tests spread over 4 days; in all training sessions the cones contained one larva each. During this period the birds became adept at foraging on the cones, and became accustomed to finding one larva per cone. Next, the birds were given six 'type-one' tests over 2 days. These were exactly the same in design as the training sessions except that (a) the birds should have by this stage formed an expectation for one larva per cone (if they were going to form any expectation at all) and (b) the tests were recorded on the organ. The final stage of the experiment was to give the birds a set of six 'type-two' tests, in which three randomly chosen trees were made into 'high-intensity' trees with three larvae per cone, while the other two trees remained as low intensity. Thus in total the birds had eighteen trials (twelve training sessions, six 'type-one' tests) with only low-intensity trees, followed by six trials with a mixture of low- and high-intensity ('type-two' tests). In all tests the position of the larvae in the cones was chosen at random. Each bird was put through a complete series of tests in turn. Between tests the test bird lived in the experimental aviary, but did not see the cones, as these were always removed at the end of a test. Before a test, the bird was deprived of food for about $20 \mathrm{~min}$, and no food other than the hidden larvae was in the aviary during the tests.

\section{Results}

In presenting the results, we will first give the averaged data for the six birds, and then discuss the variability between individuals.

(a) Hunting by expectation. The pooled data clearly show that the birds did not hunt by forming a number expectation. This hypothesis predicts that the number of larvae found per cone or cone group should be constant for highor low-intensity trees. By comparing type-one tests and the high-intensity trees in type-two tests it can be seen that these predictions are not borne out (Table I). Treating either cones or cone groups as patches the birds found more larvae in good patches than in bad patches. The data 
Table II. Experiment 1. Time Spent Hunting on (a) Each Cone and (b) Each Cone Group in Type-One Tests and Type-Two (High Intensity). Data for Six Birds Averaged

\begin{tabular}{lccc}
\hline & $\begin{array}{c}\text { Low intensity } \\
\text { mean }(N=6) \text { SD }\end{array}$ & $\begin{array}{c}\text { High intensity } \\
\text { mean }(N=6) \text { SD }\end{array}$ & $t$ \\
\hline (a) Time per cone (s) & $5.53 \pm 2.2$ & $6.52 \pm 2.7$ & $1 \cdot 83$ \\
(b) Time per group (s) & $23.105 \pm 5 \cdot 1$ & $29.63 \pm 14 \cdot 1$ & $2 \cdot 1$ \\
\hline
\end{tabular}

on time expectation are not so clear cut, but they seem to suggest that the birds spent on average longer in a good patch than in a bad patch, both when cones and when cone groups are treated as patches (Table II). However, the data do not allow us to reject conclusively the idea of hunting by time expectation.

One of the possible objections to the experimental design is the suggestion that in the typetwo tests the birds learned to treat the highand low-intensity trees as separate environments, and thus even though they had formed an expectation in the type-one tests, this was not apparent in the analysis. However, the work of Smith \& Dawkins (1971) indicates that the birds should take longer than six 5-min tests to learn to discriminate between high and low density food areas. One way to look at this possibility is to compare just the first two type-two tests with the last two type-one tests. This comparison

Table III. Data Similar to Those Presented in Tables I and II, but Only the Last Two Type-One Tests and First Two Type-Two Tests are Included. The Data show the Same Trends as the Full Analysis

\begin{tabular}{lcc}
\hline & $\begin{array}{c}\text { Low intensity } \\
\text { mean }(N=6) \mathrm{SD}\end{array}$ & $\begin{array}{c}\text { High intensity } \\
\text { mean }(N=6) \mathrm{SD}\end{array}$ \\
\hline Finds per cone & $0.26 \pm 0.03$ & $0.51 \pm 0.10$ \\
Finds per group & $0.74 \pm 0.15$ & $1.52 \pm 0.33$ \\
Time per cone (s) & $5.98 \pm 3.21$ & $6.20 \pm 2.85$ \\
Time per group (s) & $21.9 \pm 10.17$ & $29.9 \pm 12.09$ \\
\hline
\end{tabular}

(Table III) on the whole agrees with the main analysis: significantly more larvae were taken from high-intensity cones and cone groups, and there was a trend, though not statistically significant, towards spending more time on the highintensity cones and groups.

(b) Optimal foraging. In the hunting by expectation analysis, we looked at both individual cones and cone groups, while in the following analysis we look only at cone groups as patches. This is largely for technical reasons: the time spent on a cone is so short, that it was difficult to measure giving up times per cone accurately in relation to the total time on the cone. Our subjective impression of the way in which the birds foraged suggested that the cone groups rather than cones were treated as patches because, for example, a bird would frequently leave a cone for a second or two and then go straight back to it, but rarely did this with cone groups. Table IV shows that the giving-up time per cone group in the type-one tests (the poor environment) is significantly longer than the giving up time in the type-two tests (rich environment). Table $\mathrm{V}$ shows that within the type-two tests, the giving-up times for the two cone group types are not different. Thus, the data confirm two predictions of the optimal foraging model of Charnov (1973).

At this point we should discuss how we decide what constitutes a rich and poor habitat, since this is critical for the optimal foraging model. In our experiments we manipulated the food supply so that the capture rate in the second set

Table IV. Experiment 1. The Giving-Up Times (Interval Between Last Find and Leaving a Cone Group) for Poor and Rich Environments (Type-One and Type-Two Tests Respectively). The Optimal Foraging Model Predicts that the Giving-Up Time should be Shorter in the Rich Environment. The Average Rate of Prey Capture in the Rich Environment was 3.5 Prey per Minute and in the Poor Environment 2.8 Prey per Minute. Data for Six Birds Averaged

\begin{tabular}{lccc}
\hline & $\begin{array}{c}\text { Rich environment } \\
\text { mean }(N=6) \mathrm{SD}\end{array}$ & $\begin{array}{c}\text { Poor environment } \\
\text { mean }(N=6) \mathrm{SD}\end{array}$ & $t$ \\
\hline Giving-up time (s) & $11.07 \pm 4.8$ & $15.68 \pm 5.4$ & $2 \cdot 81^{*}$ \\
\hline
\end{tabular}

$* P<0.025$ (one-tailed). 
Table V. Experiment 1. Giving-Up Times for High- and Low-Intensity Cone Groups Within the 'Rich' Environment. The Optimal Foraging Model Predicts No Difference

\begin{tabular}{lccc}
\hline & $\begin{array}{c}\text { High intensity (good patch) } \\
\text { Mean }(N=6) \text { SD }\end{array}$ & $\begin{array}{c}\text { Low intensity (bad patch) } \\
\text { Mean }(N=6) \text { SD }\end{array}$ & $t$ \\
\hline Giving-up time (s) & $10.44 \pm 5 \cdot 6$ & $11 \cdot 7 \pm 6.7$ & $0 \cdot 1505$ \\
\hline
\end{tabular}

of tests was higher than in the first set $(1 \cdot 51$ prey per minute in the first, 2.47 in the second), so it seems justifiable to call the second environment 'richer'. However, in the real world, rich and poor habitats do not occur in neatly delineated packages, so the bird must have some criterion for deciding that it is in a rich environment. Presumably this involves averaging the rate of food intake over some previous short time period. We do not know the length of time over which the bird averages its capture rate to assess the quality of the habitat, but we can get some indication from our data. If we look at the transition between poor and rich environments by comparing the giving-up times in the type -one tests and the first two type-two tests, we find that there is no significant difference (type $1: 15 \cdot 68$; type $2: 14 \cdot 00, P>0 \cdot 10$ ). So apparently the birds have not 'realized' after two rich-environment tests that they are in a rich habitat, while if one compares all type-one tests with all type-two tests, there is a significant difference in giving-up times (Table IV). This suggests that a bird requires at least $10 \mathrm{~min}$ of foraging experience in an environment to assess the capture rate.

(c) Individual variation. All our discussion so far has referred to the picture obtained by averaging the data for six individuals. One should, however, bear in mind that the individual birds behaved in different ways (Table VI). All the birds were consistent in not forming a number expectation. In the case of time spent per cone group, four birds spent a longer time per patch searching in the high-intensity groups (good patches) and two spent less time per patch in the high-intensity groups. All the birds except one (GW) supported the optimal foraging model in having a shorter giving-up time in the rich than in the poor environment. This type of individual variability was also found by Smith \& Dawkins (1971) in a study of foraging in captive great tits, We cannot tell at the moment whether or not this variability is simply an artefact of using captive handraised birds. It is possible that in the wild selection acts to remove one tail of a distribution (inefficient foragers), and that by hand raising birds we are relaxing this selection. Alternatively we may not be providing an adequate environment for all individuals to learn foraging strategies, if they are learned.

\section{Experiment 2}

Experiment 1 was specifically designed to look at hunting by expectation, but the results were more consistent with Charnov's optimal foraging model. In order to test the model further, we carried out a second experiment. In this set of tests, the birds were not given extensive experience of one particular intensity of cones, but were simply put straight into an environment with three types of cone groups of different intensities. Two types of environment were used, 'rich' and 'poor'. This is likely to be more similar to the situation encountered in the real world than the set up of experiment 1 , since it must be quite unusual for a bird to encounter a long sequence of identical patch types.

\section{Procedure}

In these tests four birds were used. Each bird in turn was given a complete sequence of tests as follows. On days one and two the bird was given six 'poor-environment' tests, in which there were equal numbers (five of each) of three types of cone group with one, three and six larvae respectively. The positions of the cone groups were selected at random. On days three and four the bird was given six 'rich-environment' tests which were similar to the type one, but the environments made 'richer', by having cone groups containing three, six and twelve larvae. Recording and procedural details were otherwise exactly the same as in experiment 1.

\section{Results}

(a) Optimal foraging. The main purpose of this experiment was to compare giving-up times (i) between rich and poor environments and (ii) in different patch types within an environment. Table VII shows that even though only four birds were tested in this experiment, the average giving-up time in the rich environment is significantly shorter than that in the poor. All 
ANIMAL BEHAVIOUR, 22, 4

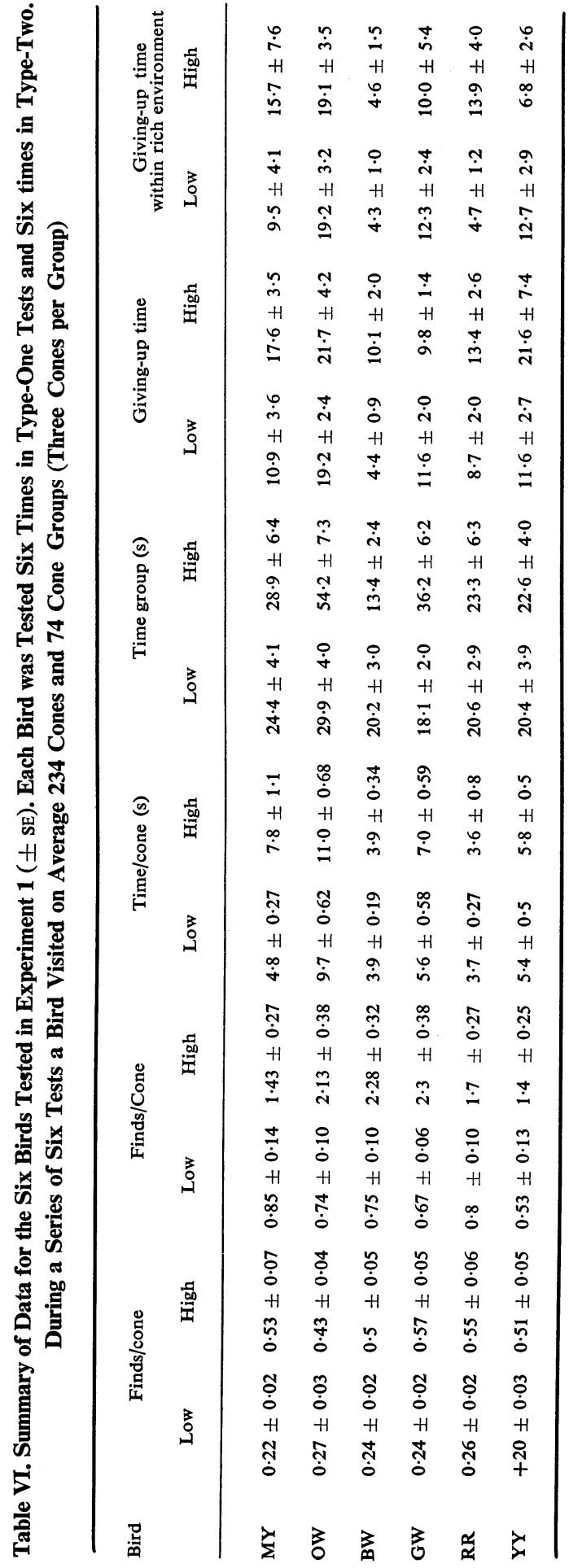


Table VII. Experiment 2. The Average Giving-up Times (For All Patch Types) of the Four Birds Tested

\begin{tabular}{lcc}
\hline & \multicolumn{2}{c}{ Giving-up time (s) } \\
\cline { 2 - 3 } Bird & Rich environment & Poor environment \\
\hline YY & 7.73 & 11.69 \\
OW & 8.11 & 15.57 \\
BW & 7.45 & 9.94 \\
GW & 6.98 & 8.30 \\
Average & $7.56^{*} \pm 0.24(\mathrm{SD})$ & $11.38^{*} \pm 1.56(\mathrm{SD})$
\end{tabular}

${ }^{*} t=2.4(6 d f) ; P<0.025$ (one-tailed).

birds showed the same trend. A one-way analysis of variance of giving-up times for the three patch types within an environment shows that there are no significant differences; this applies within both rich and poor environments (Table VIII). These results confirm those of experiment 1 and are again consistent with the optimal foraging model. Two further points worth noting are (a) that the birds took fewer larvae from cone groups of equivalent intensity when searching in the rich environment than when in the poor environment (Table IX) and (b) that the percentage of larvae taken on average from each cone group was higher in the poor environment
(22 per cent versus 15 per cent in the rich). Both of these results are consistent with the notion that the birds are more persistent (have a longer giving-up time) in the poor environment. The percentage of larvae eaten did not vary between patch types within an environment, although the optimal foraging model predicts that in a longer-term experiment where birds were allowed to deplete the environment, that there would be intensity dependent predation within an environment resulting in a constant number of larvae left in each patch (Charnov \& Orians in press).

Since the optimal foraging model predicts that the marginal rate of food intake within a patch is equal to the average intake rate for the environment, we could in theory apply a test to the model by comparing these two values to see if they are indeed equal. Although we did not measure absolute values of intake rate for each environment, we can estimate an index of relative rate of intake by looking at the relative capture rates for the different environments. The model predicts that the relative capture rates for different environments should be exactly equal to the relative values of the reciprocals of the giving up times. That is, if the capture rate in environment $A$ is twice that in $B$, the giving-up time in $B$ should be twice that in $\mathrm{A}$ (or 1/giving-up time (A) should

Table VIII. Experiment 2. Giving-Up Times (s) for Different Patch Types Within the Rich and Poor Environments. Averages for Four Birds

\begin{tabular}{lcccc}
\hline & \multicolumn{3}{c}{ Patch type } & $F$ \\
\cline { 2 - 4 } & High & Medium & Low & $(2,9 d f)$ \\
\hline Rich environment & 7.23 & 7.68 & 8.6 & 0.302 \\
Poor environment & 11.94 & 10.64 & 12.21 & 0.104 \\
\hline
\end{tabular}

Note: The terms high, medium and low patch types are explained in the text.

Table IX. Experiment 2. The Number of Larvae Found Per Patch for Different Patch Types in the Rich and Poor Environments. Averages for Four Birds

\begin{tabular}{|c|c|c|c|c|c|c|c|c|}
\hline & \multicolumn{3}{|c|}{ Finds per cone } & \multirow[b]{2}{*}{$\mathrm{F}(2,9 d f)$} & \multicolumn{3}{|c|}{ Finds per group } & \multirow[b]{2}{*}{$F(2,9 d f)$} \\
\hline & High & Medium & Low & & High & Medium & Low & \\
\hline Rich & 0.62 & 0.30 & $0 \cdot 15$ & $28 \cdot 7 * *$ & $2 \cdot 02$ & 0.90 & 0.45 & $5 \cdot 56^{*}$ \\
\hline Poor & $0 \cdot 36$ & $0 \cdot 20$ & 0.09 & $28 \cdot 8 * *$ & $1 \cdot 31$ & $0 \cdot 60$ & $0 \cdot 23$ & $20 \cdot 04 * *$ \\
\hline
\end{tabular}

$* P<0.05 ; \quad * * P<0.01$. 
be twice 1 /giving-up time (B)). We tested this prediction by combining the results of experiments 1 and 2 to give us four environments. The relative capture rates for the four environments were $2.04,1.51,1.34$ and 1.00 (from richest to poorest, in order). The reciprocals of the giving-up times for the four environments were in the ratio of $2.07: 1.42: 1.37: 1.00$. The relationship is remarkably close to that predicted by the model.

(b) Hunting by expectation. Experiment 1 enabled us to reject with some confidence the idea that the birds searched for a constant number of larvae per patch. Table IX shows that in experiment 2 also the birds did not take a constant number of larvae per patch within either environment. (Of course in this experiment the birds were not trained to expect a constant number but they could in theory form their expectation on the basis of the first few patches encountered.)

Table $X$ shows the data for time spent on each patch (treating both cones and cone groups as possible patches). In all cases there is a perfect correlation between quality of the patch type and average time spent searching in it. In the case of time per cone in the rich environment and time per group in the poor environment, the analysis of variance shows significant differences in the amount of time per patch for the different types of patch. These results strongly suggest that we were correct in tentatively rejecting the hypothesis that the birds spend a constant time per patch within a particular environment.

\section{Discussion}

To summarize our results, experiment 1 showed that even after extensive experience of an environment with uniformly low-intensity cone groups, the birds did not form a number or a time expectation. Both experiments 1 and 2 produced results that are consistent with an optimal foraging model of patch use; the giving- up time was constant for all patch types within an environment and was inversely related to the average capture rate for the environment.

Can our failure to demonstrate hunting by expectation be a function of the way we ran the experiments? We have already discussed and rejected one possible criticism; that the birds were treating the high- and low-intensity trees as separate environments within the type-two tests. Looking at the transition period between the type-one and type-two tests showed that the birds did not hunt by expectation even in their first few encounters with high-intensity cone groups. In terms of the absolute level of intensity, the birds in our experiment found $2 \cdot 3$ larvae per ten cones in the low-intensity trees while the birds studied by Gibb in the field found 2.4 larvae per ten cones. One of Gibb's more convincing pieces of evidence for hunting by expectation came from a group of patches with an intensity of twenty-six larvae per ten cones, which is comparable with our high-intensity trees with an intensity of thirty larvae per ten cones.

As we discussed earlier, one of the crucial assumptions of the optimal foraging model is that a bird can measure its average capture rate in order to assess the quality of the habitat. Recent work has shown that some species of birds are capable of measuring their rate of food intake over very short time periods (McFarland 1974; Sibley 1974), and we have argued that in our tests the birds took between 10 and $30 \mathrm{~min}$ of foraging to assess the habitat quality. Our conclusions on this point could well be misleading because of the way we carried out the tests. We tested the birds for three 5-min periods each day in the pine-cone environment, and the birds spent the rest of their time in the aviary with no pine cones. In using this arrangement, we have to assume that the birds treat the pine-cone environment as separate from their normal living environment and use special

Table X. Experiment 2. Time in Seconds Spent Searching Per Patch for Different Patch Types Within Rich and Poor Environments. Data for Four Birds Averaged

\begin{tabular}{|c|c|c|c|c|c|c|c|c|}
\hline & \multicolumn{3}{|c|}{ Time per cone } & \multirow{2}{*}{$\begin{array}{c}F \\
(2,9 d f)\end{array}$} & \multicolumn{3}{|c|}{ Time per group } & \multirow{2}{*}{$(2, \stackrel{F}{9} d f)$} \\
\hline & High & Medium & Low & & High & Medium & Low & \\
\hline Rich & $4 \cdot 87$ & $4 \cdot 23$ & $3 \cdot 63$ & $31 \cdot 01^{* *}$ & $19 \cdot 16$ & $15 \cdot 99$ & $9 \cdot 25$ & $2 \cdot 13$ \\
\hline Poor & $5 \cdot 54$ & $3 \cdot 79$ & $3 \cdot 23$ & $2 \cdot 43$ & $24 \cdot 65$ & $13 \cdot 49$ & $12 \cdot 85$ & $5 \cdot 42^{*}$ \\
\hline
\end{tabular}

$* P<0.05 ; \quad * * P<0.01$. 
values of average capture rates for the tests. There is abundant observational evidence that birds learn to associate special foraging techniques with particular habitats, so we think it not unreasonable to suppose that the birds in our tests learned to treat the pine-cone environment as a special case.

Most optimal foraging models have been based on the goal of maximizing rate of food intake (or in some cases energy gain, an index of food intake) (Charnov \& Orians in press). We have already pointed out that in the longer term an optimal forager 'aims' to maximize its fitness, and maximizing food intake is taken as a shortterm goal for convenience. Perhaps a more serious question is whether maximization should be the goal. In our particular instance, of small birds feeding in winter, the predator has to catch a food item every few seconds in order to survive (Gibb 1960), so maximization may be an appropriate goal, but other goals could be equally important. For example, in a simulation study Thompson, Vertinsky \& Krebs (1974) showed that in small birds flocking may have the effect of minimizing the risk of doing badly (going longer than a critical period without food) as well as maximizing the rate of capture. The best flock size for minimizing risk is not the same as the optimal flock size for maximizing capture rate, thus if minimizing risk is more important than maximizing efficiency, selection will not favour an optimal strategy in the sense we have defined in this paper. However, since our results are consistent with the model as it stands, we conclude that optimal foraging models of the type we have used are worth further study. Our data do not constitute a rigorous test of the model in that the predictions we tested are not precisely quantitative, but we can deduce that the concepts of marginal value and giving-up time are important in the study of patch use by predators.

\section{Acknowledgments}

We are most grateful to Dr Katharine Krebs who hand-raised the birds used in our experiments. Dolores Lauriente kindly wrote computer programs and Steve Borden built the interface for the organ. J. C. R. submitted part of this paper as a B.Sc. thesis to the University of British Columbia. We were financed by grant 67-6295 from the National Research Council of Canada to J.R.K.

\section{R E F E R E N C E S}

Charnov, E. L. (1973). Optimal foraging: some theoretical explorations. Ph.D. thesis, University of Washington.

Charnov, E. L. \& Orians, G. H. (in press). Optimal Foraging: Some Theoretical Explorations.

Croze, H. J. (1970). Searching image in carrion crows. $Z$. Tierpsychol., Beiheft 5, 85 pp.

Dawkins, R. (1972). A cheap method of recording behavioural events for direct computer access. Behaviour, 40, 162-173.

Emlen, J. M. (1973). Ecology: An Evolutionary Approach. Addison-Wesley.

Gibb, J. A. (1958). Predation by tits and squirrels on the eucosmid, Ernarmonia conicolana (Heyl). $J$. Anim. Ecol., 27, 376-396.

Gibb, J. A. (1960). Populations of tits and goldcrests and their food supply in pine plantations. Ibis, 102, 163-208.

Gibb, J. A. (1962a). L. Tinbergen's hypothesis of the role of specific search images. Ibis, 104, 106-111.

Gibb, J. A. (1962b). Tits and their food supply in English pine woods: a problem in applied ornithology. Festschr. Vogelschutzwarte Hessen, RheinlandPfalz und Saarland, 58-66.

Gibb, J. A. (1966). Tit predation and the abundance of Ernarmonia conicolana (Heyl.) on Weeting Heath, Norfolk, 1962-63. J. Anim. Ecol., 35, 43-54.

Holling, C. S. (1959). Some characteristics of simple types of predation and parasitism. Can. Ent., 91, 385-398.

Holling, C. S. (1966). The functional response of invertebrate predators to prey density. Mem. Entomol. Soc, Can., 48, 1-85.

Krebs, J. R. (1973). Behavioural aspects of predation. In: Perspectives in Ethology (Ed. by P. P. G Bateson \& P. H. Klopfer), Chapter 3. Plenum Press.

Krebs, J. R., MacRoberts, M. H. \& Cullen, J. M. (1972). Flocking and feeding in the great tit Parus major: an experimental study. Ibis, 114, 507-530.

MacArthur, R. H. \& Pianka, E. R. (1966). On optimal use of a patchy environment. Amer. Nat., 100, 603-609.

McFarland, D. J. (1974). Experimental investigation of motivational state. In: Motivational Control Systems Analysis (Ed. by D. J. McFarland). Academic Press.

Royama, T. (1970). Factors governing the hunting behaviour and food selection of the great tit Parus major. J. Anim. Ecol., 39, 619-668.

Ryan, J. C. (1973). Hunting by expectation: an experimental test. B.Sc. Thesis, University of British Columbia.

Sibly, R. M. (1975). How incentive and deficit determine feeding tendency. Anim. Behav. (in press). 
A NIMAL BEHAVIOUR, 22, 4

Simons, S. \& Alcock, J. (1971). Learning and the foraging persistence of white-crowned sparrows (Zonotrichia leucophrys). Ibis, 113, 477-482.

Smith, J. N. M. \& Dawkins, R. (1971). The hunting behaviour of individual great tits in relation to spatial variations in their food density. Anim. Behav., 19, 695-706.
Thompson, W. A., Vertinsky, I. \& Krebs, J. R. (1974). The survival value of flocking in birds; a simulation model. J. Anim. Ecol.

Tullock, G. (1971). The coal tit as a careful shopper. Amer. Nat., 105, 77-79.

(Received 19 November 1973; revised 7 March 1974; MS. number: 1272) 